06

\title{
Влияние примеси железа на электрическую проводимость кристаллов $\mathrm{LiNbO}_{3}$
}

\author{
(C) А.В. Яценко, С.В. Евдокимов
}

Крымский федеральный университет им. В.И. Вернадского,

Симферополь, Россия

E-mail: yatsenkoav@cfuv.ru

Поступила в Редакцию 19 ноября 2019 г.

В окончательной редакции 19 ноября 2019 г.

Принята к публикации 19 ноября 2019 г.

\begin{abstract}
Экспериментально исследована электрическая проводимость серии кристаллов $\mathrm{LiNbO}_{3}$, выращенных из шихты конгруэнтного состава с относительно небольшой концентрацией железа (до 0.15 mass.\% $\mathrm{Fe}_{2} \mathrm{O}_{3}$ ). Установлено, что при температурах, близких к $300 \mathrm{~K}$, основной вклад в электрическую проводимость этих кристаллов дают два типа центров с близкими значениями энергии активации. Первый тип центров ионы $\mathrm{Fe}^{2+}$ - отвечает за примесную электронную проводимость с энергией активации $(0.34 \pm 0.01) \mathrm{eV}$. Второй тип центров - поляроны малого радиуса $\mathrm{Nb}_{\mathrm{Li}}^{4+}$ с энергией активации $(0.29 \pm 0.02) \mathrm{eV}$. Показано, что для номинально беспримесных и слаболегированных „as grown“ кристаллов $\mathrm{LiNbO}_{3}$ при $T=300 \mathrm{~K}$ доминирующей является поляронная проводимость.
\end{abstract}

Ключевые слова: ниобат лития, $\mathrm{LiNbO}_{3}$, фоторефрактивный эффект, электрическая проводимость, поляроны, примеси.

DOI: 10.21883/FTT.2020.03.49009.634

\section{1. Введение}

Хорошо известно, что на оптические и электрические свойства сегнетоэлектрических кристаллов семейства $\mathrm{ABO}_{3}$ существенно влияют примеси [1,2]. Классическими представителями этого семейства являются кислородно-октаэдрические кристаллы ниобата лития $\mathrm{LiNbO}_{3}$ (НЛ) и танталата лития $\mathrm{LiTaO}_{3}$ (ТЛ). Эти материалы нашли очень широкое применение в современной оптоэлектронике, акустоэлектронике и в устройствах управления когерентным излучением оптического и ближнего инфракрасного диапазона [3].

Влияние различных примесей на удельную электрическую проводимость $\sigma$ кристаллов НЛ изучалось многими исследователями [4], причем основное внимание уделялось изучению $\mathrm{LiNbO}_{3}: \mathrm{Fe}$, так как именно в них наиболее ярко проявляется эффект фоторефракции фотоиндуцрованное изменение разности показателей преломления [1]. В кристаллах НЛ железо находится в двух состояниях $-\mathrm{Fe}^{2+}$ и $\mathrm{Fe}^{3+}$ с соответствующими объемными концентрациями $n_{\mathrm{Fe}^{2+}}$ и $n_{\mathrm{Fe}^{3+}}$. Зарядовое состояние железа в конкретном кристалле НЛ обычно характеризуется соотношением $N=n_{\mathrm{Fe}^{2+}} / n_{\mathrm{Fe}^{3+}}$. Известная на данный момент информация свидетельствует о том, что при температурах, ниже $450 \mathrm{~K}$ в температурной зависимости $\sigma(T)$ кристаллов НЛ присутствует две компоненты - ионная и электронная. Считается, что в „as grown“ кристаллах НЛ основной вклад в ионную проводимость дают ионы $\mathrm{H}^{+}$, попадающие в кристалл в процессе его роста, а электронный вклад обусловлен туннелированием термовозбужденных электронов между позициями донорных $\left(\mathrm{Fe}^{2+}\right)$ и акцепторных $\left(\mathrm{Fe}^{3+}\right)$ центров [1].
Для анализа температурной зависимости удельной электрической проводимости при наличии ионного и электронного вкладов обычно используется закон Аррениуса

$$
\begin{aligned}
\sigma & =\sigma_{\mathrm{H}}+\sigma_{e} \\
& =\left[\mu_{\mathrm{H}} \cdot N_{\mathrm{H}} \cdot \exp \left(-\frac{E_{\mathrm{H}}}{k_{o} T}\right)+\mu_{e} \cdot N_{\mathrm{eff}} \cdot \exp \left(\frac{E_{e}}{k_{o} T}\right)\right] \cdot|e|,
\end{aligned}
$$

где $|e|-$ модуль заряда электрона, $k_{o}-$ постоянная Больцмана, $\mu_{\mathrm{H}}$ и $\mu_{e}-$ подвижность протонов и электронов соответственно, $N_{\mathrm{H}}$ - объемная концентрация ионов $\mathrm{H}^{+}, N_{\mathrm{eff}}$ - эффективная объемная концентрация электронных ловушек, $E_{\mathrm{H}}$ и $E_{e}-$ энергии активации. При этом для $\mathrm{LiNbO}_{3}: \mathrm{Fe}, N_{\mathrm{eff}}=\frac{n_{\mathrm{Fe}^{2+} \cdot n_{\mathrm{Fe}} 3+}}{n_{\mathrm{Fe}^{2+}}+n_{\mathrm{Fe}} 3+} \cong n_{\mathrm{Fe}^{2+}}$, a $\mu_{\mathrm{H}}=e D_{\mathrm{H}}^{o} / k_{o} T$, где $D_{\mathrm{H}}^{o}-$ коэффициент диффузии ионов $\mathrm{H}^{+}$.

Известны попытки объяснения проводимости ,as grown“ кристаллов НЛ и ТЛ в рамках поляронного механизма [5,6], однако соответствующие выводы не являются обоснованными и корректными. В частности, примененная в [5] модуляционная методика выполнения измерений заведомо не могла обеспечить получение адекватной информации о реальном значении энергии активации электрической проводимости при температуpax близких к $300 \mathrm{~K}$ [7].

Первое систематизированное изучение удельной электрической проводимости в серии кристаллов НЛ, выращенных из расплава с добавлением $\mathrm{Fe}_{2} \mathrm{O}_{3}$ (от 0.004 до 0.75 mass.\%) было проведено в [8]. Отмечалось резкое увеличение $\sigma$ при содержании железа более, чем 
0.1 mass.\% по шихте, и явно нелинейная зависимость проводимости от концентрации железа при любой фиксированной температуре в области температур, близких к $400 \mathrm{~K}$.

Известные результаты исследования удельной электрической проводимости кристаллов $\mathrm{LiNbO}_{3}: \mathrm{Fe}$ при $T=300 \mathrm{~K}\left(\sigma_{300 \mathrm{~K}}\right)$ систематизированы в табл. 1 . Там же приведены некоторые данные для номинально беспримесных кристаллов НЛ конгруэнтного состава (CLN). Отдельно отметим, что в [12] приводится рекордно низкое значение удельной электрической проводимости кристаллов НЛ при комнатной температуре $\sigma_{r t} \cong 6 \cdot 10^{-20}(\Omega \cdot \mathrm{cm})^{-1}$. Однако эта величина была рассчитана из времени релаксации записанной голограммы и не подтверждается другими независимыми исследователями. Заметим, что после проведения термической фиксации голограммы в $\mathrm{LiNbO}_{3}: \mathrm{Fe}$ [18] время релаксации не отражает реальной картины процессов, происходящих в электронной подсистеме кристалла.

Расхождение результатов, полученных в [10], с данными других авторов для образцов с равной или сопоставимой концентрацией железа вероятно объясняется тем, что в [10] были исследованы образцы, прошедшие окислительную обработку в атмосфере сухого кислорода при температуре выше $873 \mathrm{~K}$, которая приводит к резкому уменьшению $N_{\text {eff }}[11]$.

Результаты исследования влияния общего содержания примеси железа $n_{\mathrm{Fe}}$ на темновую электрическую проводимость $\sigma_{d}$ кристаллов НЛ также неоднозначны. Так, согласно [19] при фиксированной температуре наблюдается следующая зависимость: $\sigma_{d} \propto \exp \left(a \times n_{\mathrm{Fe}}\right)$. В [12] была предложена другая форма концентрационной зависимости: $\sigma_{d} \propto \exp \left(a \times n_{\mathrm{Fe}}^{1 / 3}\right)$.

Несмотря на очень большое внимание, которое уделялось исследованию электрических свойств кристаллов НЛ с примесью $\mathrm{Fe}$, для образцов с малым контролируемым содержанием Fе информация о электрической проводимости вблизи комнатной температуры отсутствует. Это не позволяет разделить эффекты примесной и собственной проводимости кристалла. Поэтому основной целью настоящей работы было исследование температурной зависимости удельной электрической проводимости в „as grown“ кристаллах НЛ с контролируемым содержанием железа и анализ концентрационной зависимости проводимости в широком диапазоне значений $n_{\text {Fe }}$ с привлечением известных надежных данных.

\section{2. Образцы и методика эксперимента}

Для выполнения экспериментов была использована серия образцов НЛ, выращенных методом Чохральского из конгруэнтного расплава с добавлением в шихту разного количества $\mathrm{Fe}_{2} \mathrm{O}_{3}$, а также три номинально беспримесных образца НЛ конгруэнтного состава от трех разных производителей.

Для измерения электрической проводимости по постоянному току и коэффициентов оптического погло- щения использовалось то же оборудование и применялась такая же методика подготовки образцов, что и в предыдущих работах $[17,20]$. При проведении электрометрических измерений особое внимание уделялось депротонизации поверхности образцов для исключения влияния поверхностной проводимости, а также приняты дополнительные меры для повышения абсолютной точности измерений в области температур, близких к комнатной.

В качестве источника света при исследовании фототоков короткого замыкания (ф.т.к.з.) использовался мощный светодиод TDS-P005L4C11 ( $\lambda \cong 460 \mathrm{~nm}$, максимальный световой поток $\cong 150 \mathrm{~lm})$ с оптической системой, формирующей слабо расходящийся световой пучок и поляризатор ПФ-3. Плотность мощности засветки образцов измерялась прибором типа ИМО-2Н. Измерения ф.т.к.з. проводились при помощи того же электрометрического усилителя, что и для измерения электрической проводимости с модифицированной экранированной измерительной головкой, обеспечивающей минимизацию влияния на результаты измерений отраженного света.

\section{3. Анализ содержания $\mathrm{Fe}^{2+}$ в исследуемых образцах}

Для корректного сопоставления результатов исследования удельной электрической проводимости кристаллов НЛ с примесью $\mathrm{Fe}$ (в том числе - полученных другими авторами) необходимо знать не только общее содержание Fе в кристалле, но и его зарядовое состояние. В первом приближении $n_{\mathrm{Fe}^{2+}}$ можно вычислить на основании измерения коэффициента оптического поглощения на длине волны $\lambda=488 \mathrm{~nm}$ [1], однако если исследуемые образцы предварительно подвергались восстановительной термохимической обработке, то возникающая после отжига полоса оптического поглощения биполяронов накладывается на полосу поглощения $\mathrm{Fe}^{2+}[21]$, что может существенно исказить результаты такой оценки.

Известно, что при освещении кристаллов НЛ с примесью $\mathrm{Fe}$ плотность ф.т.к.з. вдоль полярного направления $j_{z}$ при фиксированной длине волны $\lambda$ пропорциональна $n_{\mathrm{Fe}^{2+}}$ и плотности мощности засветки $I$ [22]. При распространении светового потока по нормали к полярной оси и освещаемой грани тонкого кристалла величина $j_{z}$ для обыкновенного и необыкновенного луча описывается следующим образом:

$$
\left(j_{z}\right)_{o}=\beta_{o}(\lambda) \cdot I \cdot n_{\mathrm{Fe}^{2+}} \text { и }\left(j_{z}\right)_{e}=\beta_{e}(\lambda) \cdot I \cdot n_{\mathrm{Fe}^{2+}},
$$

где $\beta_{o, e}(\lambda)-$ фотовольтаические коэффициенты, зависящие от длины волны [23].

При нормальном падении светового пучка на поверхность кристалла его интенсивность (плотность мощности) $I(x)$ по мере проникновения в кристалл на глу- 
Таблица 1. Удельная электрическая проводимость и энергия активации кристаллов $\mathrm{LiNbO}_{3}$ для полярного направления $z$ и неполярного направления $y$ при $T=300 \mathrm{~K}$. Измерения по постоянному току обозначены как dс, полученные из измерения времени релаксации голограмм - как rph.

\begin{tabular}{|c|c|c|c|c|c|c|}
\hline $\begin{array}{c}\mathrm{Fe}_{2} \mathrm{O}_{3} \text { в шихте, } \\
\text { mass.\% }\end{array}$ & $N$ & Axis & $\sigma_{300 \mathrm{~K}},(\Omega \cdot \mathrm{cm})^{-1}$ & $E_{a}, \mathrm{eV}$ & Метод & Литература \\
\hline 2.0 & $0.2 \pm 0.05$ & $z$ & $\cong 2 \cdot 10^{-9}$ & $0.33 \pm 0.03$ & $\mathrm{dc}$ & {$[9]$} \\
\hline 1.0 & $0.2 \pm 0.05$ & $z$ & $\cong 1.4 \cdot 10^{-10}$ & $0.33 \pm 0.03$ & $\mathrm{dc}$ & {$[9]$} \\
\hline 0.5 & $0.2 \pm 0.05$ & $z$ & $\cong 5 \cdot 10^{-12}$ & $0.33 \pm 0.03$ & $\mathrm{dc}$ & {$[9]$} \\
\hline 0.5 & $?$ & $y$ & $\cong 8 \cdot 10^{-14}$ & & $\mathrm{dc}$ & [10] \\
\hline 0.3 & ? & $y$ & $\cong 1.1 \cdot 10^{-15}$ & & $\mathrm{dc}$ & [10] \\
\hline 0.25 & 0.1 & $z$ & $\cong 6 \cdot 10^{-15 *}$ & $\cong 0.28$ & $\mathrm{rph}$ & [11] \\
\hline 0.18 & 0.1 & $z$ & $\cong 4 \cdot 10^{-16 *}$ & & $\mathrm{rph}$ & [12] \\
\hline 0.138 & 0.03 & $z$ & $\cong 7.5 \cdot 10^{-17 *}$ & $\cong 0.28$ & rph & [13] \\
\hline 0.07 & $?$ & $z$ & $\cong 1.9 \cdot 10^{-16}$ & $0.33 \pm 0.03$ & $\mathrm{dc}$ & {$[14]$} \\
\hline 0.02 & 0.2 & $z$ & $6 \cdot 10^{-20 *}$ & & $\mathrm{rph}$ & {$[12]$} \\
\hline CLN & & $z$ & $\cong 1 \cdot 10^{-15}$ & $0.39 \pm 0.02$ & $\mathrm{dc}$ & {$[15]$} \\
\hline CLN & & $z$ & $\approx 3 \cdot 10^{-16}$ & $\approx 0.25$ & $\mathrm{dc}$ & [16] \\
\hline CLN & & $z$ & $(7.4 \pm 1.5) \cdot 10^{-17}$ & $0.33 \pm 0.04$ & $\mathrm{dc}$ & {$[17]$} \\
\hline
\end{tabular}

При ме чан ие. * Данные получены при т. н. „комнатной“ температуре.

бину $x$ уменьшается в соответствии с законом БугераЛамберта

$$
I_{o, e}(x)=I_{o}\left(1-R_{o, e}\right) \exp \left(-\alpha_{o, e} x\right),
$$

где $R_{o, e}-$ коэффициенты отражения, $\alpha_{o}$ и $\alpha_{e}-$ коэффициенты оптического поглощения обыкновенного и необыкновенного луча на соответствующей длине волны $\lambda$.

Комбинируя (2) и (3), несложно получить выражение для описания фотовольтаического тока $I_{\mathrm{ph}}$ в короткозамкнутом оптически плотном кристалле толщиной $d$

$$
\begin{aligned}
I_{\mathrm{ph}} & =a \beta_{o, e}(1-R) I_{o} n_{\mathrm{Fe}^{2+}} \int_{0}^{d} \exp \left(-\alpha_{o, e} \cdot x\right) d x \\
& =a \beta_{o, e}(1-R) I_{o} n_{\mathrm{Fe}^{2+}} \frac{\left[1-\exp \left(-\alpha_{o, e} \cdot d\right)\right]}{\alpha_{o, e}},
\end{aligned}
$$

где $a$ - длина полярной грани. Выражение (4) и было применено нами для определения $n_{\mathrm{Fe}^{2+}}$ в данной серии кристаллов.

Результаты измерения оптической плотности исследованных образцов $\alpha_{o}$ и $\alpha_{e}$ приведены на рис. 1 и в табл. 2. Из рис. 1 видно, что значение $\alpha_{o}$ растет примерно пропорционально общему содержанию Fе в образце. Это обстоятельство является косвенным свидетельством того, что данные кристаллы не проходили дополнительные термохимические обработки.

Использованное в расчетах значение фотовольтаического коэффициента $\left(\beta_{e}\right)_{460}$ для длины волны $\lambda=460 \mathrm{~nm}$ было получено коррекцией значения $\beta_{e}$, полученного в [24] для $\lambda=488 \mathrm{~nm}:\left(\beta_{e}\right)_{488}=$ $=(6.5 \pm 1.2) \cdot 10^{-33} \mathrm{~m}^{3} \mathrm{~V}^{-1}$ согласно спектральной зависимости $\beta_{e}(\lambda)$ [23] (увеличение в 1.36 раза). Результаты измерения ф.т.к.з. при $I_{o}=(5.0 \pm 0.2) \mathrm{mW} / \mathrm{cm}^{2}$

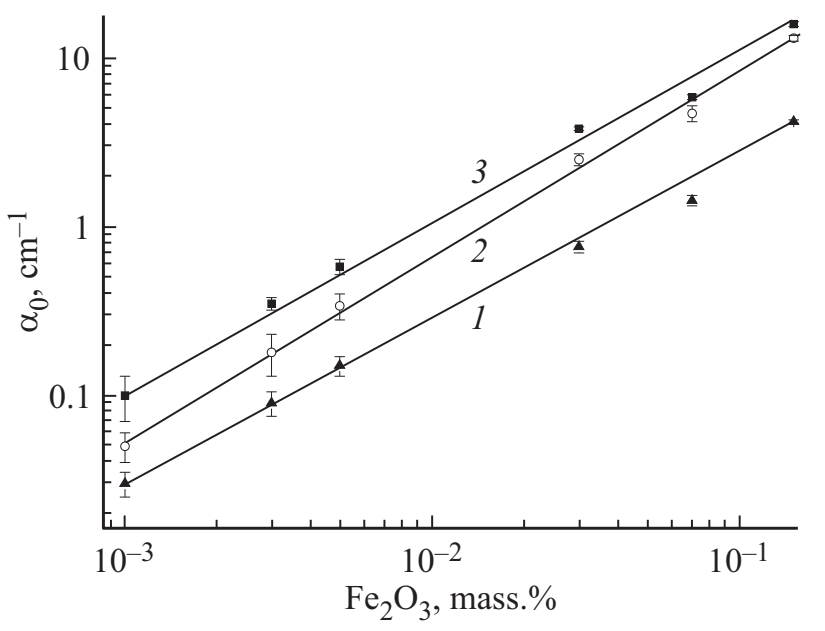

Рис. 1. Зависимость коэффициента поглощения обыкновенного луча от содержания $\mathrm{Fe}_{2} \mathrm{O}_{3}$ в шихте на длине волны $\lambda$ : (1) $-625 \mathrm{~nm}$; (2) $-525 \mathrm{~nm}$; (3) $-465 \mathrm{~nm}$.

и результаты расчета $n_{\mathrm{Fe}^{2+}}$ в исследованных образцах представлены в табл. 2.

\section{4. Исследование температурной зависимости электрической проводимости}

Измерение температурной зависимости электрической проводимости проводилось не только для образцов, представленных в табл. 2, но и еще для двух номинально беспримесных кристаллов НЛ конгруэнтного состава с целью уточнения значения удельной электрической проводимости и энергии активации при температуре $T=300 \mathrm{~K}$. На рис. 2-4 представлены эксперименталь- 
Таблица 2. Параметры исследуемых образцов, результаты измерений ф.т.к.з. $\left(I_{\mathrm{ph}}\right)_{z}\left(I_{\mathrm{ph}}\right.$ и полученные значения $n_{\mathrm{Fe}} 2+$

\begin{tabular}{c|c|c|c|c|c|c}
\hline № & $\begin{array}{c}\mathrm{Fe}_{2} \mathrm{O}_{3} \text { в шихте, } \\
\text { mass.\% }\end{array}$ & $d, \mathrm{~mm}$ & $\left(\alpha_{e}\right) 465 \mathrm{~nm}, \mathrm{~cm}^{-1}$ & $\left(I_{\mathrm{ph}}\right)_{z}, \mathrm{pA}$ & $n_{\mathrm{Fe}^{2+}, 10^{17} \mathrm{~cm}^{3}}$ & $N$ \\
\hline 1 & $\mathrm{CLN}$ & 4.99 & $0.13 \pm 0.04$ & $\sim 0.1$ & $<0.1$ & $?$ \\
2 & 0.003 & 2.82 & $0.32 \pm 0.05$ & $0.92 \pm 0.08$ & $0.72 \pm 0.15$ & $0.10 \pm 0.02$ \\
3 & 0.005 & 2.78 & $0.62 \pm 0.06$ & $3.22 \pm 0.15$ & $2.0 \pm 0.4$ & $0.17 \pm 0.03$ \\
4 & 0.03 & 3.21 & $3.38 \pm 0.15$ & $11.1 \pm 0.3$ & $14 \pm 3$ & $0.20 \pm 0.04$ \\
5 & 0.07 & 6.42 & $5.26 \pm 0.26$ & $11.5 \pm 0.4$ & $22 \pm 4$ & $0.13 \pm 0.03$ \\
6 & 0.15 & 2.06 & $14.5 \pm 0.5$ & $21.7 \pm 0.5$ & $60 \pm 12$ & $0.17 \pm 0.03$
\end{tabular}

ные результаты, полученные для образцов № 2, № 5 и № 6, а также результаты их оптимальной аппроксимации выражением (1). На этих же рисунках отмечена температура $T^{\prime}$, при которой электронный и ионный вклад в проводимость одинаковы.

Для образца № 6 значение энергии активации, отвечающее за низкотемпературную область $E_{2}$ составляет величину $(0.34 \pm 0.01) \mathrm{eV}$, что в пределах погрешно-

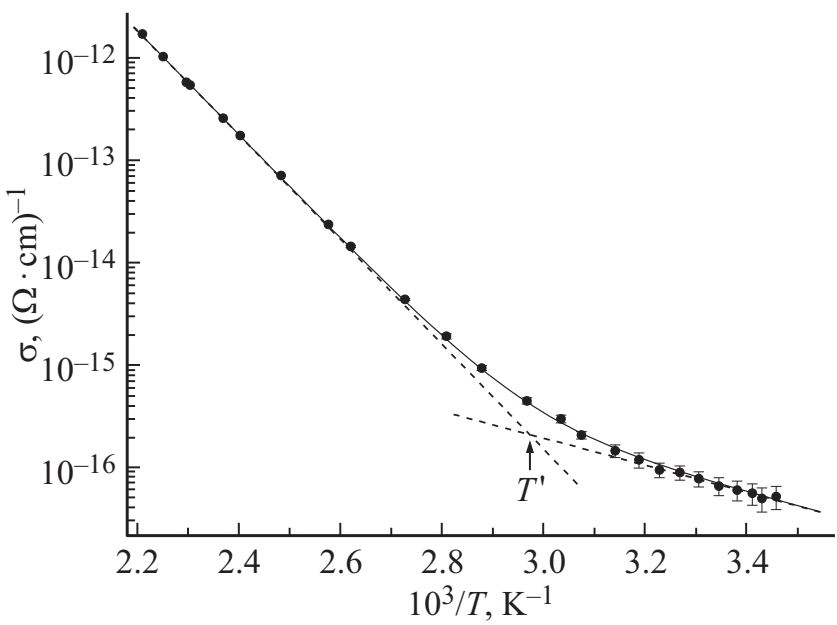

Рис. 2. Температурная зависимость удельной электрической проводимости образца № 2 (0.003 mass.\% $\mathrm{Fe}_{2} \mathrm{O}_{3}$ по шихте).

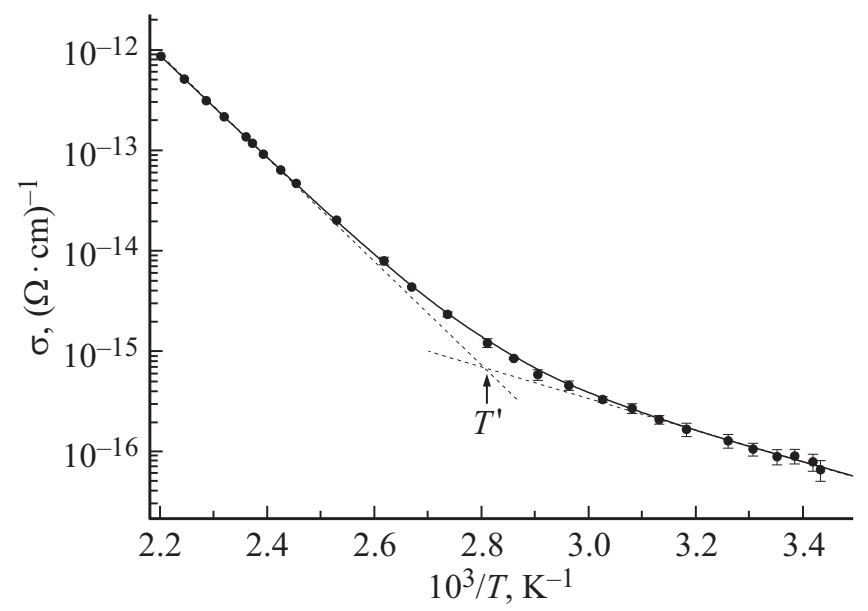

Рис. 3. Температурная зависимость удельной электрической проводимости образца № 5.

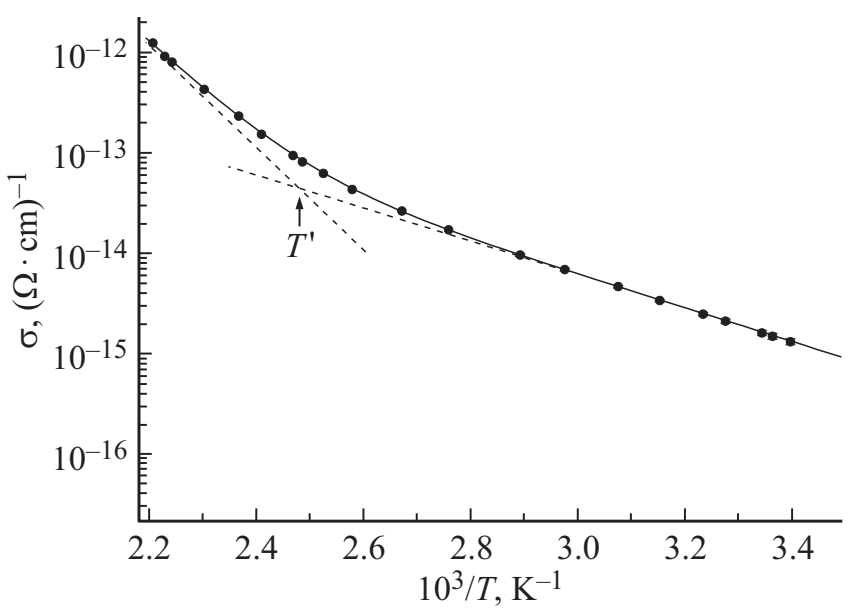

Рис. 4. Температурная зависимость удельной электрической проводимости образца № 6.

сти совпадает со значением, полученным в [9], а для трех номинально беспримесных и слабо легированных образцов соответствующее значение лежит в пределах $(0.27-0.30) \mathrm{eV}$. В то же время у образца № 5 это значение близко к последнему: $(0.30 \pm 0.01) \mathrm{eV}$.

Исходя из этого была проведена еще одна аппроксимация экспериментальных данных для образца № 5, учитывающая три вклада в проводимость, характеризуемых фиксированными энергиями активации $E_{1}=1.05 \mathrm{eV} ; E_{2}=0.34 \mathrm{eV}$ и $E_{3}=0.29 \mathrm{eV}$

$$
\begin{aligned}
\sigma(T)=\frac{C_{1}}{T} \cdot \exp ( & \left.-\frac{E_{1}}{k_{o} T}\right)+C_{2} \exp \left(-\frac{E_{2}}{k_{o} T}\right) \\
& +C_{3} \exp \left(-\frac{E_{3}}{k_{o} T}\right),
\end{aligned}
$$

где $C_{1}, C_{2}, C_{3}-$ соответствующие коэффициенты пропорциональности.

Оказалось, что в этом случае аппроксимирующая зависимость характеризуется меньшим среднеквадратичным отклонением от экспериментальных данных, чем для зависимости типа (1), т.е. является более точной. Значения параметров, отвечающих оптимальной аппроксимации экспериментальных результатов выражением (5) для исследованных образцов, приводятся в табл. 3. На рис. 5 приводится зависимость удельной 
Таблица 3. Параметры, описывающие электрическую проводимость кристаллов НЛ с примесью железа

\begin{tabular}{c|c|c|c|c|c|c|c}
\hline № & $E_{1}, \mathrm{eV}$ & $\begin{array}{c}C_{1}, \\
(\Omega \cdot \mathrm{cm})^{-1} \cdot \mathrm{K}\end{array}$ & $E_{2}, \mathrm{eV}$ & $\begin{array}{c}C_{2}, \\
(\Omega \cdot \mathrm{cm})^{-1}\end{array}$ & $E_{3}, \mathrm{eV}$ & $\begin{array}{c}C_{3}, \\
(\Omega \cdot \mathrm{cm})^{-1}\end{array}$ & $\begin{array}{c}1000 / T^{\prime}, \\
\mathrm{K}^{-1}\end{array}$ \\
\hline 1 & $1.05 \pm 0.01$ & $510 \pm 30$ & & & $0.28 \pm 0.02$ & $(4.3 \pm 1.5) \cdot 10^{-12}$ & $\cong 2.98$ \\
2 & $1.05 \pm 0.01$ & $370 \pm 30$ & & & $0.29 \pm 0.02$ & $(5.1 \pm 1.5) \cdot 10^{-12}$ & $\cong 2.97$ \\
3 & $1.06 \pm 0.01$ & $250 \pm 30$ & & & $0.28 \pm 0.02$ & $(4.9 \pm 1.5) \cdot 10^{-12}$ & $\cong 2.95$ \\
4 & $1.05 \pm 0.01$ & $300 \pm 30$ & & & & & \\
5 & $1.05 \pm 0.01$ & $230 \pm 30$ & 0.34 & $(5 \pm 3) \cdot 10^{-12}$ & 0.29 & $(6.5 \pm 2.0) \cdot 10^{-12}$ & $\cong 2.90$ \\
6 & $1.06 \pm 0.01$ & $210 \pm 30$ & $0.34 \pm 0.01$ & $(8.3 \pm 1) \cdot 10^{-10}$ & & & $\cong 2.86$ \\
\end{tabular}

электрической проводимости исследованных кристаллов при $T=300 \mathrm{~K}\left(\sigma_{300 \mathrm{~K}}\right)$ от их коэффициента оптического поглощения $\alpha_{e}$ при $\lambda=465 \mathrm{~nm}$.

\section{5. Анализ и обсуждение результатов}

Данные, представленные в табл. 3 и на рис. 5 наглядно свидетельствуют о том, что при концентрациях железа, отвечающих $\left(\alpha_{e}\right)_{465} \leq 3.2 \mathrm{~cm}^{-1}$ влияние примеси $\mathrm{Fe}$ на электрическую проводимость в пределах погрешности отсутствует. Так как у исследованных образцов значения $N$ различаются более, чем в 2 раза, целесообразно рассмотреть нормированную величину: $\sigma_{300 \mathrm{~K}} / N_{\text {eff }}[22]$. Значения $N_{\text {eff }}$ рассчитывались по данным табл. 2 в предположении полного вхождения железа в кристалл в процессе синтеза. Полученная зависимость $\sigma_{300 \mathrm{~K}} / N_{\mathrm{eff}}$ от среднего расстояния между ионами железа $\left\langle r_{\mathrm{Fe}-\mathrm{Fe}}\right\rangle=\left(n_{\mathrm{Fe}}\right)^{-1 / 3}$ изображена на рис. 6. На этом же рисунке приводятся некоторые известные данные из табл. 1 для кристаллов НЛ с большой концентрацией железа. Отдельно (звездочкой) представлено значение удельной проводимости для образца № 5, отвечающее выделенному вкладу носителей с энергией активации $E_{2}=0.34 \mathrm{eV}$.

Наглядно видно, что в области высоких значений $n_{\text {Fe }}$ экспериментальные результаты достаточно хорошо

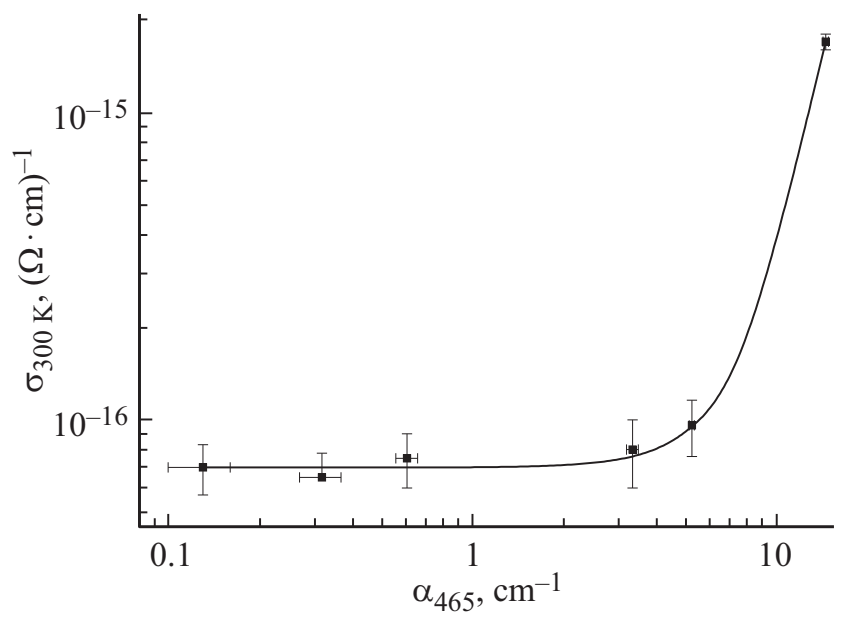

Рис. 5. Зависимость $300 \mathrm{~K}$ от коэффициента оптического поглощения для исследованных кристаллов $\mathrm{LiNbO}_{3}$ : Fe. Аппроксимация сплайном.

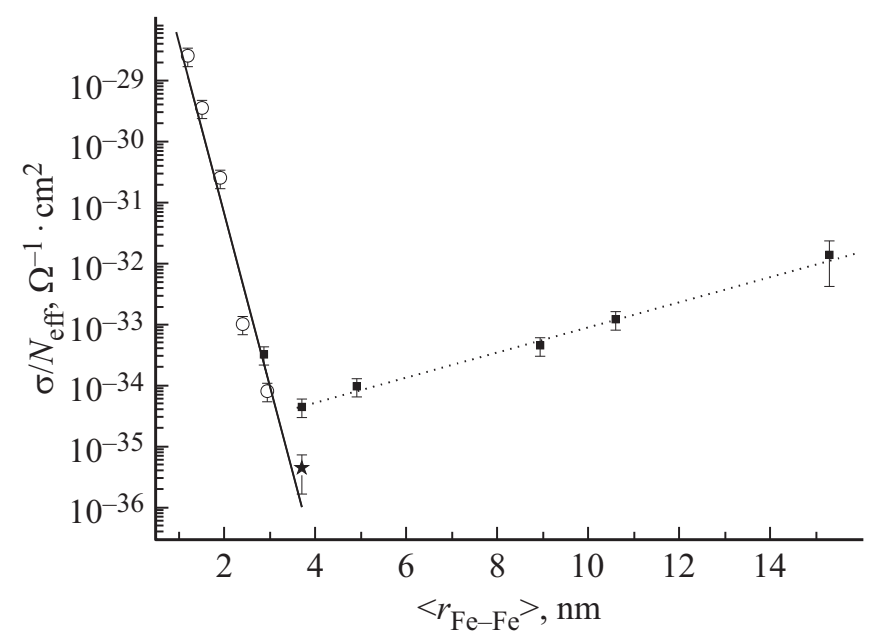

Рис. 6. Нормированная электрическая проводимость $\sigma / N_{\text {eff }}$ как функция среднего расстояния между ионами железа в кристаллах НЛ. Результаты $[9,11,13]$ представлены открытыми символами. Сплошная линия - аппроксимация выражением (6).

аппроксимируются экспоненциальным законом, предложенным в [12] :

$$
\sigma_{300 \mathrm{~K}} / N_{\mathrm{eff}} \propto \exp \left(-a\left\langle r_{\mathrm{Fe}-\mathrm{Fe}}\right\rangle\right)
$$

где $a \cong 6.5 \mathrm{~nm}^{-1}$. Аналогичный тип зависимости электрической проводимости кристаллов НЛ от концентрации ионов железа был установлен и в [22] - для диапазона значений $\left\langle r_{\mathrm{Fe}-\mathrm{Fe}}\right\rangle$ от $1.25 \mathrm{~nm}$ до $2 \mathrm{~nm}$.

Зависимость нормированной удельной проводимости от общей объемной концентрации железа $n_{\mathrm{Fe}}$ изображена на рис. 7. Подобный вид зависимости характерен для проводимости диэлектрических композитов с металлическими включениями и описывает явление перколяционной проводимости. Согласно перколяционному подходу экспериментальные данные в области больших значений $n_{\mathrm{Fe}}$ могут быть аппроксимированы степенным законом

$$
\sigma_{300 \mathrm{~K}} / N_{\mathrm{eff}} \propto b\left(n_{\mathrm{Fe}}-n_{0}\right)^{p},
$$

где $b$ и $p-$ константы, а $n_{0}-$ т.н. порог перколяции [25]. Из оптимальной аппроксимации следует, что $n_{0} \cong 2.2 \cdot 10^{18} \mathrm{~cm}^{-3}, p \cong 4.68$. 


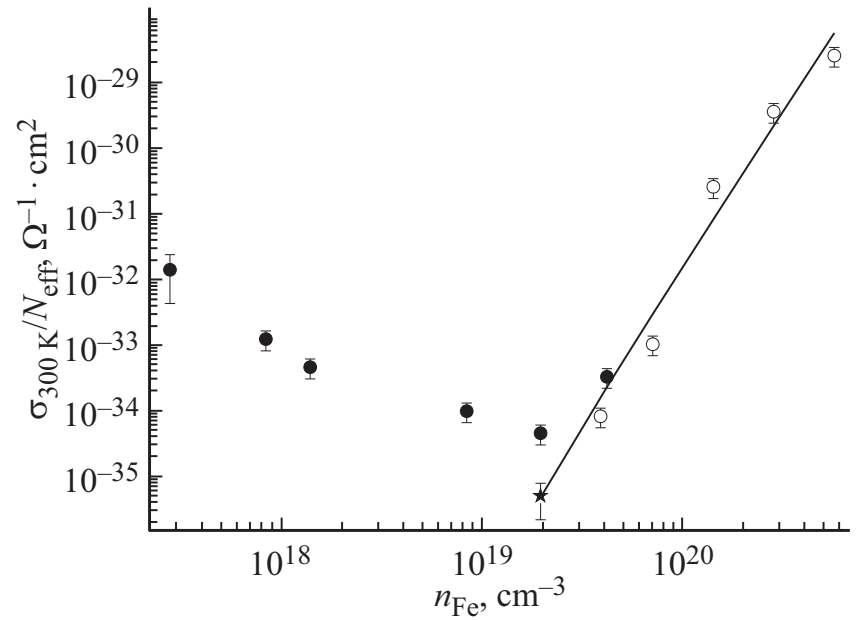

Рис. 7. Нормированная электрическая проводимость как функция объемной концентрации ионов железа в кристаллах НЛ. Результаты $[9,11,13]$ представлены открытыми символами. Сплошная линия - аппроксимация выражением (7).

Сравнение качества аппроксимации экспериментальных данных выражениями (6) и (7) проводилось по следующему критерию:

$$
S=\left[\frac{1}{n} \sum_{i=1}^{n}\left[\left(\frac{w_{i}-f_{i}}{w_{i}}\right)^{2}+\left(\frac{w_{i}-f_{i}}{f_{i}}\right)^{2}\right]\right]^{1 / 2},
$$

где $w_{i}$ и $f_{i}$ - соответственно расчетное и экспериментальное значение нормированной проводимости в $i$-й точке, $n=7$ - количество экспериментальных точек. Было установлено, что для экспоненциального закона $S_{\min }=2.11$, а для степенного $-S_{\min }=1.34$.

Значение $\sigma_{300} \mathrm{~K}$ для трех исследованных здесь номинально беспримесных кристаллов НЛ конгруэнтного состава заведомо не подвергавшихся послеростовому отжигу в атмосфере сухого кислорода (от разных изготовителей $)$ лежит в пределах $(6-10) \cdot 10^{-17}(\Omega \cdot \mathrm{cm})^{-1}$, a $E_{3} \cong 0.29 \mathrm{eV}$. В этот диапазон укладывается и значение $\sigma_{300 \mathrm{~K}}$, полученное в [19] для CLN методом TWM (two wave mixing) $-8.3 \cdot 10^{-17}(\Omega \cdot \mathrm{cm})^{-1}$. Отметим, что недавно проведенные исследования электрических свойств кристаллов НЛ, выращенных из расплава с флюсом $\mathrm{K}_{2} \mathrm{O}$, показали, что по мере приближения состава к стехиометрическому значение $\sigma_{300}$ к существенно уменьшается - при сохранении значения энергии активации $E_{3} \cong 0.29 \mathrm{eV}[26]$.

Наши результаты однозначно свидетельствуют о том, что в области температур вблизи комнатной кроме примесной проводимости $\left(E_{2} \cong 0.34 \mathrm{eV}\right)$ в кристаллах НЛ присутствует еще один процесс, характеризующийся энергией активации $E_{3} \cong 0.29 \mathrm{eV}$, который может быть описан в рамках поляронного механизма.

Совокупность известных и новых экспериментальных данных свидетельствует в пользу того, что в номинально беспримесных и слабо легированных кристаллах НЛ в области температур, близких к комнатной, доминирующей является именно поляронная, а не примесная проводимость. Среднее расстояние между антиструктурными ионами $\mathrm{Nb}_{\mathrm{Li}}^{5+}$ в кристалле НЛ конгруэнтного состава составляет величину $1.74 \mathrm{~nm}$, поэтому в слаболегированном НЛ после термоактивации донорного центра $\mathrm{Fe}^{2+}$ электрон с максимальной вероятностью захватывается мелкими ловушками $\mathrm{Nb}_{\mathrm{Li}}^{5+}$ с образованием полярона малого радиуса (ПМР) $\mathrm{Nb}_{\mathrm{Li}}^{4+}$. Далее происходит термовозбуждение образовавшегося ПМР и последующеее туннелирование электрона по мелким ловушкам - вплоть до захвата более глубокой ловушкой $\mathrm{Fe}^{3+}$. При этом с уменьшением $n_{\mathrm{Fe}}$ должна увеличиваться вероятность обратной рекомбинации исходных донорных центров, что объясняет специфический закон для концентрационной зависимости проводимости. Подобный механизм ранее уже обсуждался в рамках модели транспорта фотоиндуцированного электрона по двум типам ловушек в процессе возникновения фоторефракции [1]. Отметим, что в последнее время достаточно активно обсуждается роль ПМР в фоторефрактивном эффекте [27-29].

В рамках существования поляронного механизма находит объяснение и резкое уменьшение проводимости образцов НЛ с примесью Fе после проведения сильного оксидирования [12] - при этом не только резко уменьшается соотношение $N$, но и уменьшается объемная концентрация водорода. Вполне вероятно, что в процессе окислительного отжига номинально беспримесных кристаллов НЛ водород покидает образец в нейтральном состоянии - с захватом электронов от существующих в образце ПМР $\mathrm{Nb}_{\mathrm{Li}}^{4+}$ в соответствии с реакцией $2 \mathrm{H}^{+}+2 \mathrm{Nb}_{\mathrm{Li}}^{4+} \rightarrow \mathrm{H}_{2}+2 \mathrm{Nb}_{\mathrm{Li}}^{5+}$. В этом случае после выполнения окислительного отжига должно наблюдаться пропорциональное уменьшение ионного и электронного вклада в электрическую проводимость.

\section{6. Заключение}

На основании экспериментального исследования серии номинально беспримесных кристаллов НЛ конгруэнтного состава и образцов НЛ, легированных железом, можно сделать следующие выводы. Установлено, что для „as grown“ кристаллов НЛ легированных железом с содержанием менее 0.03 mass. $\% \mathrm{Fe}_{2} \mathrm{O}_{3}$ (по шихте) в области температур, близких к комнатной, примесная электронная проводимость более чем на порядок меньше, чем поляронная проводимость.

Показано, что значения энергий термической активации примесных ионов $\mathrm{Fe}^{2+}$ и ПМР $\mathrm{Nb}_{\mathrm{Li}}^{4+}$ несколько различаются и равны $(0.34 \pm 0.01) \mathrm{eV}$ и $(0.29 \pm 0.02) \mathrm{eV}$ соответственно.

Делается предположение, что в процессе окислительного отжига в сухом кислороде номинально беспримесных кристаллов НЛ происходит не только их депротонизация, но и пропорциональное уменьшение объемной концентрации ПМР. 


\section{Конфликт интересов}

Авторы заявляют, что у них нет конфликта интересов.

\section{Список литературы}

[1] T.R. Volk, M. Wöhlecke. Lithium Niobate. Defects, photorefraction and ferroelectric switching. Springer-Verlag, Berlin (2008). 250 p.

[2] N. Ramadass. Mater. Sci. Eng. 36, 231 (1978).

[3] L. Arizmendi. Phys. Status. Solidi. A 201, 253 (2004).

[4] L. Kovács, K. Polgar. In: Properties of Lithium Niobate / Ed. K.K. Wong, INSPEC. The Institution of Electrical Engineers, London, UK (2002). 429 p.

[5] Q. Wang, S. Leng, Y. Yu. Phys. Status Solidi B 194, 661 (1996).

[6] A. El-Bachiri, F. Bennani, M. Boulessamti. Spectrosc. Lett. 47, 374 (2014).

[7] А.С. Притуленко, А.В. Яценко, С.В. Евдокимов. Кристаллография 60, 293 (2015).

[8] I.B. Barkan, M.V. Entin, S.I. Marennikov. Phys. Status Solidi A 44, K91 (1977).

[9] K. Brands, M. Falk, D. Haertle, T. Woike, K. Buse. Appl. Phys. В 91, 279 (2008).

[10] Г.Г. Здоровцев, Ю.М. Карпец, В.А. Лебедев. Фундаментальные исследования 9, 1512 (2014).

[11] Y. Yang, I. Nee, K. Buse, D. Psaltis. In: OSA Tops. Vol. 62. Photorefractive effects, materials and devices / Ed. D. Nolte, G. Salamo. (2001). P. 144-151.

[12] I. Nee, M. Müller, K, Buse, E. Krätzig. J. Appl. Phys. 88, $4282(2000)$.

[13] Y. Yang, I. Nee, K. Buse, D. Psaltis. Appl. Phys. Lett. 78, 4076 (2001).

[14] С.В. Евдокимов, А.В. Яценко. ФТТ 48, 317 (2006).

[15] И.Ш. Ахмадуллин, В.А. Голенищев-Кутузов, С.А. Мигачев, С.П. Миронов. ФТТ 40, 1307 (1998).

[16] W. Bollmann, M. Gernand. Phys. Status Solidi A 9, 301 (1972).

[17] А.В. Яценко, М.Н. Палатников, Н.В. Сидоров, А.С. Притуленко, С.В. Евдокимов. ФТТ 57, 932 (2015).

[18] J.J. Amodei, D.L. Staebler. Appl. Phys. Lett. 18, 540 (1971). H. Vormann, G. Weber, S. Kapphan, E. Kratzig. Solid State Commun. 40, 543 (1981).

[19] D. Kip, J. Hukriede, E. Krützig. Phys. Status Solidi A 168, R3 (1998).

[20] А.В. Яценко, А.С. Притуленко, С.В. Ягупов, Д.Ю. Сугак, И.М. Сольский. ФТТ 60, 521 (2018).

[21] J. Koppitz, O.F. Shirmer, A.I. Kuznetsov. Europhys. Lett. 4, 1055 (1987).

[22] M. Falk, J. Japs, T. Woike, K. Buse. Appl. Phys. B 87, 119 (2007).

[23] H.G. Festl, Hertel P. Krätzig E., R. von Baltz. Phys. Status Solidi B 113, 157 (1982).

[24] J. Jermann, J. Otten. Opt. Soc. Am. B 10, 2085 (1993).

[25] D. Stauffer, A. Aharony. Introduction to percolation theory. Taylor \& Francis, London (1992). 181 p.
[26] А.В. Яценко, С.В. Евдокимов, М.Н. Палатников, Н.В. Сидоров. ФТТ 61, 1270 (2019).

[27] O.F. Shirmer, M. Imlau, C. Merschjann. Phys. Rev. B 83, 165106 (2011).

[28] M. Imlau, H. Badorreck, C. Merschjann. Appl. Phys. Rev. 2, 040606 (2015)

[29] L. Vittadello, M. Bazzan, A. Danielyan, E. Kokanyan, L. Guibert, M. Aillerie. J. Phys. Commun. 2, 125003 (2018).

Редактор Ю.Э. Китаев 